\title{
Imprinted photonic crystal nanocavity composed of inversely tapered air holes for highly sensitive optical sensor
}

\author{
Kenichi Maeno ${ }^{1}$, Shoma Aki ${ }^{1}$, Kazuo Satoh², Shunichi Murakami², Yusuke Sando², Yusuke Kanaoka², \\ Kenji Sueyoshi' ${ }^{1}$ Hideaki Hisamoto ${ }^{1}$, Tatsuro Endo ${ }^{1,3 *}$ \\ 1 Osaka Prefecture University, Sakai, Osaka 599-8531, Japan., \\ 2 Osaka Research Institute of Industrial Science and Technology(ORIST), Osaka 594-1157, Japan \\ 3 JST PRESTO, Tokyo, Japan \\ *endo@chem.osakafu-u.ac.jp
}

\begin{abstract}
Polymer-based photonic crystal nanocavity (PCN) composed of tapered and inversely tapered air holes for optical sensing application was successfully fabricated. Polymer-based PCN fabricated by using nanoimprint lithography (NIL) is a promising device for highly-sensitive optical sensor. From the view point of sensing application, the efficiency of light confinement in PCN is a significant factor. However, polymer-based PCN possessed low efficiency caused of low refractive index of polymer material. In this research we found that the shape of air holes composing PCN contributed to the confinement efficiency. We fabricated PCNs composed of cylindrical, tapered, and inversely-tapered air holes by using NIL technique. As an optical measurement result, the inversely tapered one showed the strongest light confinement and two times higher $Q$ value than that of tapered one.
\end{abstract}

Key words: Photonic crystal nanocavity, Optical sensor, Nanoimprint lithography, inverse taper

\section{Introduction}

Photonic crystal nanocavity (PCN) is an optical resonator capable to confine the light of specific wavelength strongly into nanoscale based on Bragg diffraction derived from wavelengthdimensional periodic nanohole array. This optical phenomenon induces strong light-matter interaction within nanoscale and has applied to various applications, such as ultra-small light sources [1], optical manipulation systems [2], and also optical biosensors [3]. For these applications, quality $(Q)$ factor which designates the efficiency of light confinement is a significant parameter. The value of $Q$ factor is defined as $Q=\lambda_{0} / \Delta \lambda$. Here $\lambda_{0}$ and $\Delta \lambda$ indicate resonant wavelength and full width of half maximum of resonant spectrum, respectively. We previously reported polymer-based PCN fabricated on metal substrate which can prevent the light leakage to the base substrate, and successfully observed visible light confinement into PCN [4]. However, the $Q$ value was very low, below one hundred, because of the light leakage to air from photonic crystal slab caused by low refractive index of polymer material. Therefore, suppression of this leakage is important point to improve $Q$ value.
Here, we focused on the fact that the smaller air hole radius leads the higher average refractive index ( $n_{\text {ave. }}$ ) of the slab because of the high occupancy of polymer material. This fact means the $n_{\text {ave. }}$ value is "locally" controllable by tuning air hole radius along the depth direction (Fig. 1). In this study, we fabricated three types of polymer-based PCNs composed of different shape of air holes, cylindrical, tapered and inversely tapered shapes. $Q$ values were evaluated by spectrometric analysis.

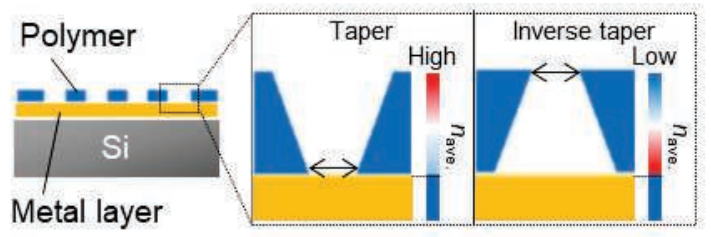

Fig. 1. Schematic illustration of $n_{\text {ave }}$ controlled by tuning air hole radius along the depth direction.

\section{Experimental}

Ideal $Q$ values (Qideal) of polymer-based $P C N s$ composed of triangle lattice cylindrical, tapered, or inversely tapered air holes were calculated by using simulation analysis (FDTD solutions). The lattice constant was $300 \mathrm{~nm}$, and the air hole radius was $80 \mathrm{~nm}$. In the case of tapered 
or inversely tapered shape, the maximum and the minimum air hole radii were $90 \mathrm{~nm}$ and 70 $\mathrm{nm}$, respectively.

These structures were fabricated according to the procedure described below (Fig. 2). Firstly, the pillar structure of cylindrical, tapered, or inversely tapered shape was fabricated on Si by using electron beam lithography and dry etching process (Fig. 1 (a)). Secondly, the structure was transferred to UV resin (NOA 81) as a $2^{\text {nd }}$ mold by using UV-NIL (Fig. 2 (b)). Thirdly, water-soluble polymer (PVA: poly(vinyl alcohol)) was coated on $2^{\text {nd }}$ mold and dried up under $80^{\circ} \mathrm{C}$. Then, a sacrificial mold was obtained by demolding (Fig. 2 (c)). Finally, the polymer resin (SU-8 2000.5) was coated on the sacrificial mold and attached on metal substrate. After dissolving of sacrificial mold, polymerbased PCN composed of cylindrical or tapered air holes was fabricated (Fig. 2 (d) upper). Only the case of inversely tapered shape, the tapered structure of SU-8 resin was attached on silicone rubber (PDMS: poly (dimethyl siloxane)). After dissolving of sacrificial mold and flipping PDMS, the structure of SU-8 resin was transferred on metal substrate, and the PCN composed of inversely tapered air holes was fabricated (Fig. 2 (d) lower).

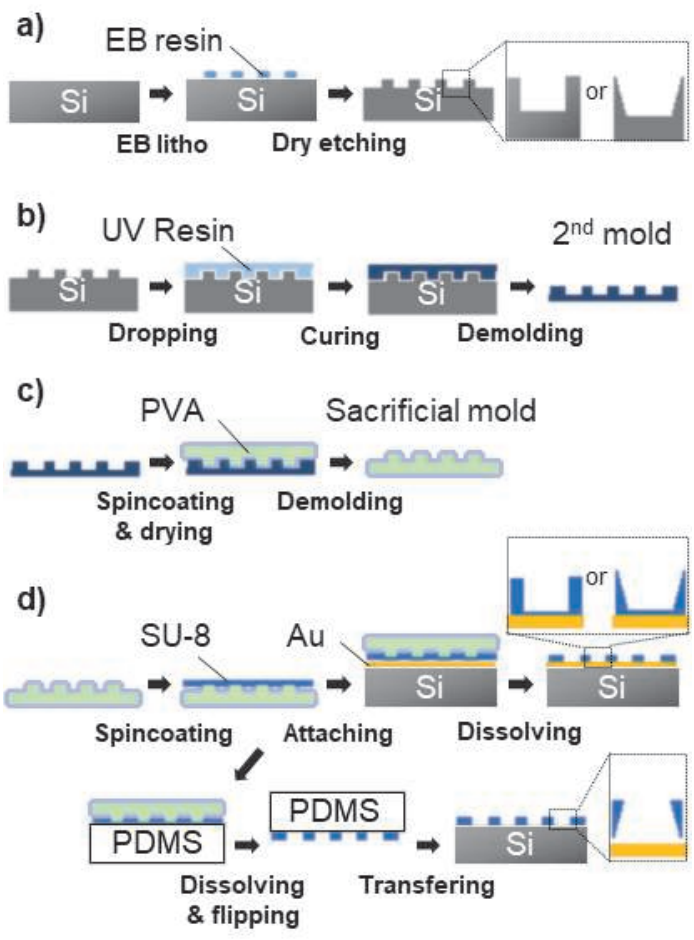

Fig. 2. Fabrication procedure of polymer-based $\mathrm{PCN}$.

Experimental $Q$ values ( $Q \exp$ ) of fabricated PCNs were evaluated as described below. A broadband white light $(400-800 \mathrm{~nm})$ was irradiated from the edge of photonic crystal structure. Then, the light of resonant wavelength was confined in PCN. The confined light was measured by using spectrometer, and Qexp was calculated by $\lambda_{0}$ and $\Delta \lambda$ of measured spectrum.

\section{Results and discussion}

Measured spectrum of PCN composed of inversely tapered air holes was shown in Fig. 3. In Fig. 3, very sharp peak was observed at wavelength of $580 \mathrm{~nm}(\Delta \lambda=3 \mathrm{~nm})$.

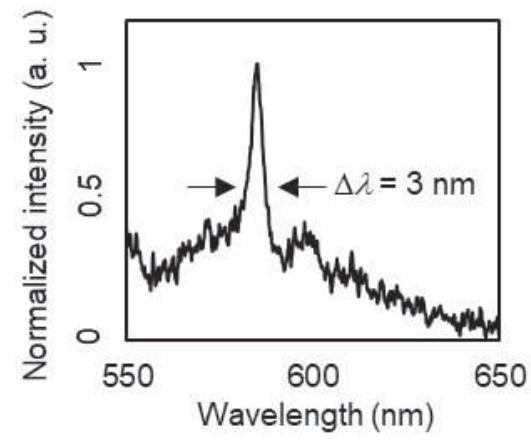

Fig. 3. Measured spectrum of PCN composed of inversely tapered air holes.

Evaluated Qideal and Qexp of PCNs were summarized in Table 1. The PCN composed of inversely tapered air holes showed the highest $Q$ value, approximately two times higher than that of tapered one. These results suggested that the shape of air hole was a significant factor for improvement of $Q$ values.

Table 1: Qideal and Qexp of each PCNs.

\begin{tabular}{|c|c|c|c|}
\hline & Taper & Cylinder & $\begin{array}{c}\text { Inverse } \\
\text { taper }\end{array}$ \\
\hline$Q_{\text {idel }}$ & 159 & 202 & 232 \\
\hline$Q_{\exp }$ & 95 & 150 & 203 \\
\hline
\end{tabular}

Acknowledgement

This work is supported by JST PRESTO.

\section{References}

[1] Y. Takahashi, Y. Inui, M. Chihara, T. Asano, R. Terawaki, and S. Noda, Nature 498, 7455, 470 474 (2013); doi: 10.1038/nature12237.

[2] T. van Leest and J. Caro, Lab on a chip, 13, 22, 4358-4365 (2013); doi: 10.1039/C3LC50879J.

[3] S. Hachuda, S. Otsuka, S. Kita, T. Isono, M. Narimatsu, K. Watanabe, Y. Goshima, and T. Baba, Optics Express 21, 10, 12815-12821 (2013); 10.1364/OE.21.012815.

[4] K. Maeno, J. Sun, S. Aki, K. Sueyoshi, H. Hisamoto, T. Endo, proceeding of IMCS 2016, A472. 